Doug Geisler, Eva K. Grebel, and Dante Minniti, eds.

\title{
Globular Clusters around Galaxies in Groups
}

\author{
Cristiano Da Rocha \& Claudia Mendes de Oliveira \\ Instituto de Astronomia, Geofísica e Ciências Atmosféricas, \\ Universidade de São Paulo, Av. Miguel Stefano 4200, 04301-904, São \\ Paulo - SP, Brazil \\ Michael Bolte \\ UCO/Lick Observatory, Department of Astronomy and Astrophysics, \\ University of California, Santa Cruz, California 95064, USA
}

\section{Bodo L. Ziegler}

Universitätssternwarte Göttingen, Geismarlandstr. 11, 37083 Göttingen, Germany

Thomas H. Puzia

Universitätssternwarte München, Scheinerstr. 1, D-81679 München, Germany

\begin{abstract}
.
We have obtained deep photometry of NGC 1199 (in HCG 22) and NGC 6868 (in the Telescopium group). Both galaxies are the optically brightest galaxies of their groups. Our analysis of $B$ and $R$ images taken with the Keck II and the VLT/ESO telescopes, detected a population of globular clusters around both galaxies, with total specific frequencies $S_{N}=1.7 \pm 0.6$ for NGC 1199 and $S_{N}=1.3 \pm 0.6$ for NGC 6868. The color distributions of the globular cluster systems shows bimodal peaks centered at $(B-R)_{0}=1.13 \pm 0.10$ and $1.42 \pm 0.10$ (NGC 1199) and $(B-R)_{0}=1.12 \pm 0.10$ and $1.42 \pm 0.10$ (NGC 6868).
\end{abstract}

\section{Introduction}

The properties of extragalactic globular cluster systems (GCS) - specific frequency, color distribution and radial profile shape - may be expected to vary as a function of the environment of the host galaxy.

The main goal of this work is to determine the properties of the globular cluster systems of two galaxies in small groups: the central elliptical galaxy in HCG 22 (NGC 1199) and the suspected merger in the center of the Telescopium group (NGC 6868) (Hansen, Jørgensen \& Nørgaard-Nielsen, 1991).

We assume distance moduli of $(m-M)_{V}=32.6$ and 32.1 for NGC 1199 and NGC 6868 respectively as determined from surface-brightness fluctuations (Tonry et al. 2001). 


\section{Observations and Data Reduction}

The images of HCG 22 were obtained with the Keck II telescope, using LRIS, in $B$ and $R$ with total exposure times of 720 and 630 seconds and average seeing of 0.77 and 0.74 arcsec respectively. The NGC 6868 images were obtained with the VLT/ESO, using FORS1, in $B$ and $R$ with total exposure times of 900 and 810 seconds and average seeing of 0.76 and 0.73 arcsec respectively. Images in $B$ and $R$ of similar depth were obtained centered on a position $10^{\prime}$ from the galaxy center to be used as a control field for background subtraction.

The light profile of the bright galaxies was modeled with ELLIPSE/STSDAS and we used SExtractor (Bertin \& Arnouts, 1996) for detection and photometry of the faint objects.

\section{Specific Frequency and Radial Profile Modeling}

In order to calculate the specific frequency, the observed number counts were corrected by the lost area due to masks and unobserved regions. We fit a Gaus$\operatorname{sian}\left(M_{V}=-7.6\right.$ and $\sigma=1.18$, Drenkhahn \& Richtler, 1999), to the bright, measured GCLF and extrapolate the number of GC's over all magnitudes in our systems. Those counts were used as boundary conditions to integrate the radial profile models and we obtained the total number of objects in the GCS's.

We tried two kinds of radial profiles, a "core model" profile $\left(\rho=\rho_{0}\left(r_{c}^{\alpha}+\right.\right.$ $\left.r^{\alpha}\right)^{-1}$ ) with the core radius proposed by Forbes et al. (1996) $\left(r_{c}(k p c)=-(0.62 \pm\right.$ $\left.0.1) \cdot M_{V}-11.0\right)$ and a power-law profile $\left(\rho \propto r^{\alpha}\right)$.

For NGC 1199 the slope of the power-law profile is -2.4 . This slope is very steep, leading to unrealistic values. Using this slope for the outer region of the "core model" we estimated a $S_{N}=1.7 \pm 0.6$, which is smaller than the mean value found for a typical small group elliptical (Harris, 1991, gives 2.6 \pm 0.5 ), but still in agreement within the error bars. For NGC 6868 the slope of the powerlaw is -1.4 . We estimated $S_{N}=1.4 \pm 0.6$ with this profile. The "core model", with the same slope, gives us $S_{N}=1.3 \pm 0.6$. Those estimates are a factor of two smaller than typical indicating a poor system around this galaxy. The radial profiles for both galaxies and the best-fit power-law and "core model" are shown in Figure 1.

\section{Color Distribution}

The KMM test (Ashman, Bird \& Zepf, 1994) was used to estimate the parameters of the color distributions. We have detected bimodal populations for both galaxies with $100 \%$ confidence level. The peaks are located at $(B-R)_{0}=$ $1.13 \pm 0.10$ and $1.42 \pm 0.10$ for NGC 1199 and $(B-R)_{0}=1.12 \pm 0.10$ and $1.42 \pm 0.10$ for NGC 6868 . Using Reed, Harris \& Harris (1994) color-metallicity relation, we have $[\mathrm{Fe} / \mathrm{H}]=-1.45 \pm 0.23 \mathrm{dex}$ and $-0.55 \pm 0.23$ dex for the blue and red peaks of NGC 1199, respectively and $[\mathrm{Fe} / \mathrm{H}]=-1.48 \pm 0.23 \mathrm{dex}$ and $-0.55 \pm 0.23 \mathrm{dex}$ for the blue and red peaks of NGC 6868 , respectively.

Acknowledgments. This work is supported by FAPESP PhD. grant No. 96/08986-5 and Pronex NExGAL. 


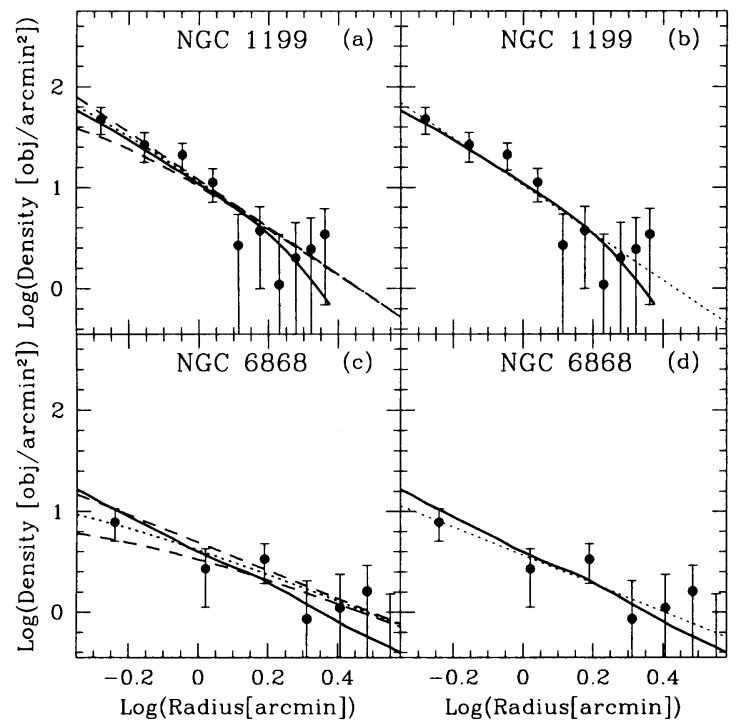

Figure 1. Radial profiles of globular cluster candidates. Panel (a) shows the radial profile of GC's around NGC 1199 with the "core model" profile (dotted line) and its upper and lower limits (dashed lines) and the galaxy light in the B band (continuous line) overplotted. Panel (b) shows the the power-law profile (dotted line) overplotted for NGC 1199. Panels (c) and (d) show the same information of panels (a) and (b), respectively, for the GCS around NGC 6868.

\section{References}

Ashman, K. M., Bird, C. M. \& Zepf, S. E. 1994, AJ, 108, 2348

Bertin, E. \& Arnouts, S. 1996, A\&A, 117, 393

Drenkhahn, G. \& Richtler, T. 1999, A\&A, 349, 877

Forbes, D. A., Franx, M., Illingworth, G. D. \& Carollo, C. M. 1996, ApJ, 467, 126

Hansen, L., Jørgensen, H. E. \& Nørgaard-Nielsen, H. U. 1991, A\&A, 243, 49 Harris, W. E. 1991, ARA\&A, 29, 543

Reed, L. G., Harris, G. L. H. \& Harris, W. E. 1994, AJ, 107, 555

Tonry, J. L., Dressler, A., Blakeslee, J. P., Ajhar, E. A., Fletcher, A. B., Luppino, G. A., Metzger, M. R. \& Moore, C. B. 2001, ApJ, 546, 681 